\title{
Intuitionistic Fuzzy in Spline Curve/Surface
}

Mohammad Izat Emir Zulkifly and Abd Fatah Wahab*

School of Informatics and Applied Mathematics, Universiti Malaysia Terengganu, Malaysia.

*Corresponding Author: fatah @umt.edu.my

\section{Article history :}

Received 6 October 2014

Accepted 20 February 2015

\section{GRAPHICAL ABSTRACT}

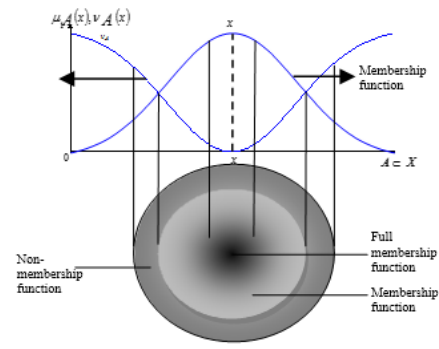

\section{ABSTRACT}

The uncertainty data problem with intuitionistic information is difficult to deal with through the methodology or approach available. This is because the existing fuzzy geometric modeling can only solve the fuzzy data problem that is intuitionistic and fuzzy complex in nature as the data have information that is incomplete boundary value (less clear and ambiguous), have meaning and truth value of the range, a lot of value logic values as well as having vague distribution and indistinct. Furthermore, the operation is characteristically minimum or maximum and image together with the range are obscure and complex. To solve this problem, a new model with a redefinition of the control points which characterized by three important components of intuitionistic fuzzy is developed. These control points will be blended with the basic functions of spline to generate several models in the form of intuitionistic fuzzy spline curve / surface and can be easily understood. With the intuitionistic concept and its relations, then the relevant data can be translated through the production of these models.

Keywords: intuitionistic fuzzy, intuitionistic fuzzy spline, control points, fuzzy complex, fuzzy geometry modeling, spline curve / surface

(c) 2015 Penerbit UTM Press. All rights reserved http://dx.doi.org/10.11113/mjfas.v11n1.343

\section{INTRODUCTION}

The basic concept of fuzzy set theory was introduced by Zadeh [1] in 1965 to solve the problem of uncertainty that occur in daily life. Several years after that, Atanassov [2] has introduced a new theory based on fuzzy set theory known as fuzzy intuitionistic in 1986 involving the degrees of membership and nonmembership. By using the theory of Atanassov [2], some obscure theorem intuitionistic will be developed in the context of fuzzy geometric modeling with basic characteristics. The concept of intuitionistic fuzzy numbers appearing in fuzzy concept will be discussed and examined for the production of basic theorems intuitionistic new fuzzy. Through the development of this theorem, the data points will be defined as Intuitionistic Fuzzy Control Point (IFCP). These control points are blended together with spline basis functions in model geometry to produce new fuzzy spline hybrid models known as Intuitionistik Fuzzy Bezier Model (IFBM). The Intuitionistic Fuzzy Spline Model (IFSM) can be applied in the design of geometric objects. Moreover, this model can also be applied in the problem of data uncertainty that is often occur in science and engineering such as the reconstruction of an object, mental models, real-time tracking and remote scanning. Moreover, this model can be used for data analysis in Geographic Information Systems (GIS) for spatial data.

\section{BASIC CONCEPTS}

The theory of fuzzy sets was introduced by Zadeh [1] in 1965 to solve problems involving uncertainty. The theory of fuzzy sets are defined as

$$
A=\left\{\left(x, \mu_{A}(x)\right) \mid x \in X\right\}
$$

which $\mu_{A}(x)$ represents the degree of membership of the element $x$ to the set $A$ in the interval $[0,1]$. For intuitionistic fuzzy sets, suppose universal set $X$ and set of object $x$ which have the fuzzy valued logic, therefore the intuitionistik fuzzy set $A$ in $D$ is $\mu_{A}: D \rightarrow I \times I$ a function which for each $x \in D$ exists $\mu_{A}(x)$ fuzzy valued logic of $x$ in $A \subset X$ and $v_{A}(x) \operatorname{logic}$ value for $x$ is not a member in $A \subset X$ which

$0 \leq \mu_{A}(x)+\mu_{A^{C}}(x) \leq 1$

Hence, intuitionistik fuzzy set $A$ in $X$ is defined as an object as follows [2,3]:

$A=\left\{\left\langle x, \mu_{A}(x), v_{A}(x)\right\rangle \mid x \in X\right\}$

where the function

$\mu_{A}: X \rightarrow[0,1]$

and

$v_{A}: X \rightarrow[0,1]$

defined degrees of membership and non-membership degree of the element $x \in X$, respectively, and for each $x \in X$

$0 \leq \mu_{A}(x)+v_{A}(x) \leq 1$ 
Obviously, each ordinary fuzzy set can be written as follow:

$$
\left\{\left\langle x, \mu_{A}(x), 1-\mu_{A}(x)\right\rangle x \in X\right\}
$$

Different from fuzzy sets introduced by Zadeh [1], the sum of $\mu_{A}$ and $v_{A}$ is not necessarily equal to 1 and its value can be obtained and known through intuitionistic. This situation is very useful when the data obtained is not complete. Then,

$$
\pi_{A}(x)=1-\mu_{A}(x)-v_{A}(x)
$$

where $\pi_{A}(x)$ is known as degree of uncertainty or hesitation, and it represents the information contained therein. Therefore, based on the definition of intuitionistic fuzzy set above, three important components will be the basis of the approach in this study, namely:

$$
\begin{aligned}
& A^{\mu}=\left\{x, \mu_{A}(x) \mid \mu_{A}(x) \in[0,1]\right\} \\
& A^{v}=\left\{x, v_{A}(x) \mid v_{A}(x) \in[0,1]\right\} \\
& A^{\pi}=\left\{x, \pi_{A}(x) \mid \pi_{A}(x) \in[0,1]\right\}
\end{aligned}
$$

where equation (9) is degree of membership, equation (10) is non-membership degree and equation (11) is degree of uncertainty or hesitation.

Therefore, based on the concept and theory of intuitionistic fuzzy introduced by Atanassov [2], some theorem will be developed in the context of fuzzy geometric modeling for construction of Intuitionistic Fuzzy Control Point (IFCP) which is fundamental in the construction of the model.

\section{MODEL CONSTRUCTION METHOD}

Intuitionistic spline model in the context of geometric modeling results when each coefficient geometry spline model redefined through intuitionistic fuzzy approach until produced a form of control points. Next, with the blending process and recursive process, the curve / surface is generated. The control points is defined as

$$
P=\left\{P_{i}\right\}_{i=0}^{n-1}
$$

for each $P_{i} \in P$ exists $\mu_{p}\left(P_{i}\right), v_{p}\left(P_{i}\right)$ and $\pi_{p}\left(P_{i}\right)$ in $[0$, 1]. Based on control points in equation (12) and three important components in equation (9), (10) and (11), three new control points are derived as follows.

$$
\begin{aligned}
& P^{\mu}=\left\{P_{i}, \mu_{P}\left(P_{i}\right)\right\} \\
& P^{v}=\left\{P_{i}, v_{P}\left(P_{i}\right)\right\} \\
& P^{\pi}=\left\{P_{i}, \pi_{P}\left(P_{i}\right)\right\}
\end{aligned}
$$

where equation (13) is membership component control points, equation (14) is non-membership component control points and equation (15) as an uncertainty component control points.

\section{Intuitionistic Fuzzy Bezier Model}

Let fuzzy spline introduced by Wahab et al. [4]

$S(t)=\sum_{i=0}^{n} P_{i} B(t)$ with $0 \leq t \leq 1$

known as fuzzy spline model with $P_{i}=\left\{P_{0}, P_{1}, \ldots, P_{n}\right\}$ is fuzzy control points and $B(t)$ is basis spline function. With the new IFCP, hence

$\tilde{P}_{i}^{d}=\left\{\tilde{P}_{0}^{d}, \tilde{P}_{1}^{d}, \ldots, \tilde{P}_{n-1}^{d}\right\}$

is introduced until the general Intuitionistic Fuzzy Bezier Model Curve (IFBMC) is written as

$\tilde{S}_{B}(t)=\sum_{i=0}^{n} \tilde{P}_{i}^{d} B_{i}^{n}(t)$ with $0 \leq t \leq 1$

with

$\widetilde{S}_{B}(t)=\sum_{i=0}^{n} \widetilde{P}_{i}^{\mu} B_{i}^{n}(t)$ with $0 \leq t \leq 1$

$\tilde{S}_{B}(t)=\sum_{i=0}^{n} \widetilde{P}_{i}^{v} B_{i}^{n}(t)$ with $0 \leq t \leq 1$

$\tilde{S}_{B}(t)=\sum_{i=0}^{n} \widetilde{P}_{i}^{\pi} B_{i}^{n}(t)$ with $0 \leq t \leq 1$

with $\widetilde{P}_{i}^{d}$ is the intuitionistic fuzzy control points where $d$ stands for types of control points and $B(t)$ is the basis spline function for Bezier. For Intuitionistic Fuzzy Bezier Model Surface, the general form is written as

$\widetilde{S}_{B}(u, v)=\sum_{i=0}^{n} \sum_{j=0}^{m} \widetilde{P}_{i j}^{d} B_{i}^{n}(u) B_{j}^{m}(v)$ with $0 \leq u \leq 1$ and

$0 \leq v \leq 1$

with

$\tilde{S}_{B}(u, v)=\sum_{i=0}^{n} \sum_{j=0}^{m} \tilde{P}_{i j}^{\mu} B_{i}^{n}(u) B_{j}^{m}(v)$ with $0 \leq u \leq 1$ and

$0 \leq v \leq 1$

$\tilde{S}_{B}(u, v)=\sum_{i=0}^{n} \sum_{j=0}^{m} \tilde{P}_{i j}^{v} B_{i}^{n}(u) B_{j}^{m}(v)$ with $0 \leq u \leq 1$ and

$0 \leq v \leq 1$

$\widetilde{S}_{B}(u, v)=\sum_{i=0}^{n} \sum_{j=0}^{m} \widetilde{P}_{i j}^{\pi} B_{i}^{n}(u) B_{j}^{m}(v)$ with $0 \leq u \leq 1$ and

$0 \leq v \leq 1$ 


\section{DATA DEFUZZIFICATION METHOD}

For the defuzzification of intuitionistic uncertainty data, the defuzzification is as in Figure 1.

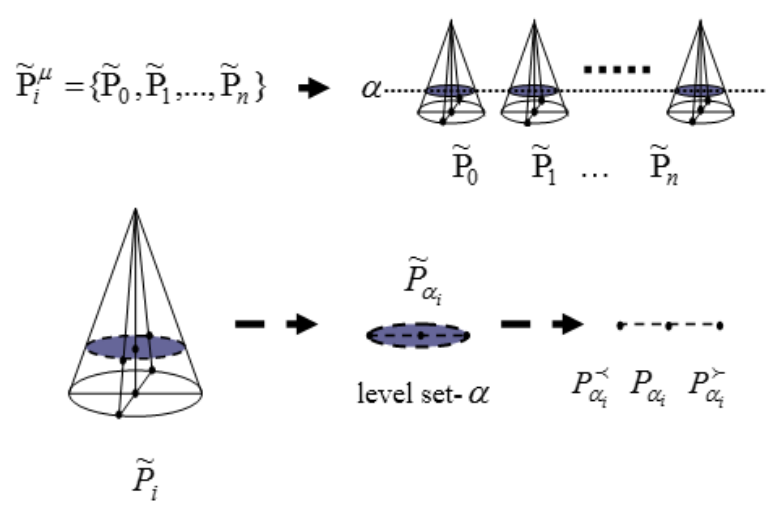

Figure 1. Intuitionistic fuzzy control point in interval $<P_{i}^{\prec}, P_{i}^{\mu}, P_{i}^{\succ}>$ in a level set of $[0,1]$.

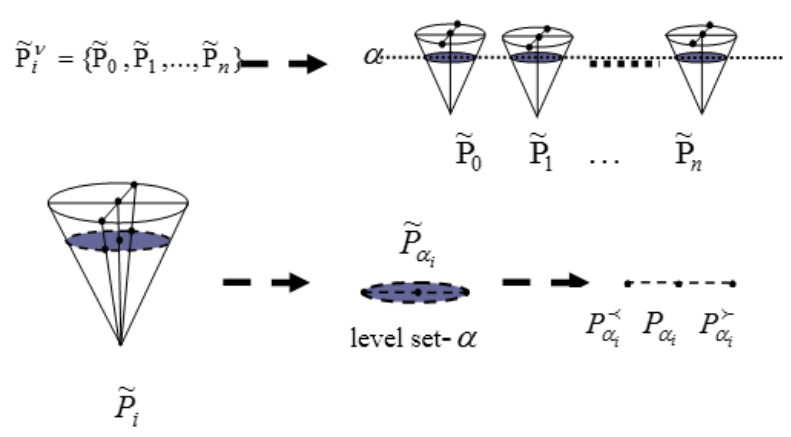

Figure 2. Intuitionistic fuzzy control point in interval $<P_{i}^{\prec}, P_{i}^{v}, P_{i}^{\succ}>$ in a level set of $[0,1]$.

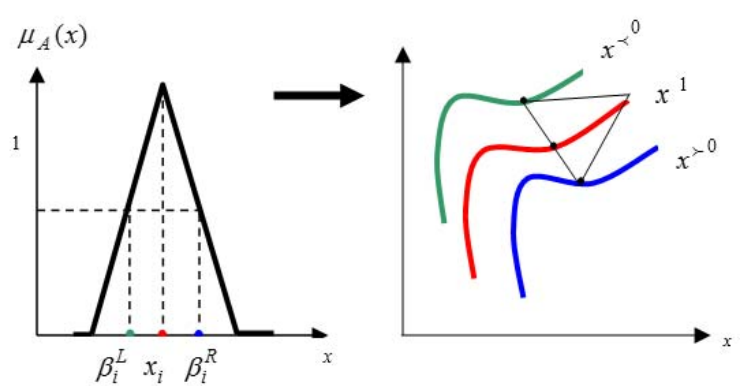

Figure 3. Mapping of fuzzy value for $\mu_{A}(x)$

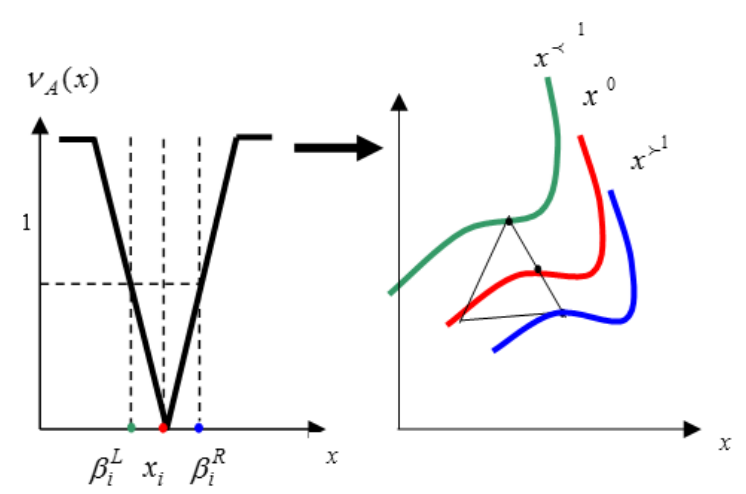

Figure 4. Mapping of fuzzy value for $v_{A}(x)$

\section{CONCLUSION}

Based on the defuzzification process from Figure 1 until Figure 4, the problem of intuitionistic uncertainty data can be solved through intuitionistic fuzzy geometric models. The advantage of this model is its capability to translate its data into a form of intuitionistic fuzzy model or a curve/surface that is continuous and smooth, and maintains ambiguity of information in the form of a simple and easy to understand by the data analyst. The method and the resulting model will be able to contribute to the field of fuzzy modeling techniques. As this model is geometric, it is needed in the areas of pre-designed surface geometry of an object in a particular industry in Malaysia.

\section{ACKNOWLEDGEMENTS}

This work was supported by Research Management Center (RMC) Universiti Malaysia Terengganu (UMT) and Kementerian Pengajian Tinggi (KPM) through ERGS Research Grant No. 55099.

\section{References}

[1] L.A. Zadeh, Fuzzy Sets, Information and Control. 8 (1965) 338.

[2] K.T. Atanassov, Intuitionistic Fuzzy Sets, Fuzzy Sets and Systems. 20 (1986) 87.

[3] K.T. Atanassov, Intuitionistic Fuzzy Sets, Springer PhysicaVerlag, Berlin, 1999.

[4] A.F. Wahab, J.M. Ali, A.A. Majid, A.O.M. Tap, Fuzzy Geometric Modelling, $6^{\text {th }}$ International Conference on Computer Graphics, Imaging and Visualization. China (2009) 227-232. 